\title{
La question du citoyen actif : les conservateurs britanniques face à la Révolution française
}

\section{Emma Vincent MacLeod}

\section{(2) OpenEdition \\ 1 Journals}

\section{Édition électronique}

URL : https://journals.openedition.org/ahrf/1908

DOI : 10.4000/ahrf.1908

ISSN : 1952-403X

Éditeur :

Armand Colin, Société des études robespierristes

\section{Édition imprimée}

Date de publication : 1 décembre 2005

Pagination : 47-72

ISSN : 0003-4436

\section{Référence électronique}

Emma Vincent MacLeod, « La question du citoyen actif : les conservateurs britanniques face à la

Révolution française ", Annales historiques de la Révolution française [En ligne], 342 | octobre-décembre 2005, mis en ligne le 15 décembre 2008, consulté le 23 avril 2022. URL : http://

journals.openedition.org/ahrf/1908; DOI : https://doi.org/10.4000/ahrf.1908

Ce document a été généré automatiquement le 23 avril 2022.

Tous droits réservés 


\title{
La question du citoyen actif : les conservateurs britanniques face à la Révolution française
}

\author{
Emma Vincent MacLeod
}

\begin{abstract}
« To enable men to act with the weight and character of a people, and to answer the ends for which they are incorporated into that capacity, we must suppose them [...] to be in that state of habitual social discipline, in which the wiser, the more expert, and the more opulent conduct, and by conducting enlighten and protect, the weaker, the less knowing, and the less provided with the goods of fortune. When the multitude are not under this discipline, they can scarcely be said to be in civil society. »
\end{abstract}

1 Dès avant les années 1980, la manière dont les conservateurs britanniques ont réagi à la Révolution française a suscité des travaux importants. H.T. Dickinson s'est intéressé à l'idéologie ${ }^{2}$, Gayle Trusdel Pendleton à la propagande ${ }^{3}$, Nancy Murray à l'Église d'Angleterre ${ }^{4}$; les associations loyalistes ont attiré l'attention d'Austin Mitchell, d'Eugene Black et Donald Ginter ${ }^{5}$; enfin, les Volontaires ont fait l'objet d'une étude de J.R. Western ${ }^{6}$. À cela s'ajoute bien évidemment l'impressionnant corpus de travaux publiés sur les Réflexions d'Edmund Burke concernant la Révolution française ${ }^{7}$. Il a fallu attendre 1985, avec un chapitre du British Radicalism and the French Revolution de H. T. Dickinson, pour que ces livres épars soient appréhendés de façon synthétique. Depuis lors, comme l'ont suggéré Mark Philp et Kevin Gilmartin ${ }^{8}$, les chercheurs travaillant sur les milieux conservateurs dans les années 1790 ont répondu à l'appel d'E. P. Thompson - déjà pris en compte par les spécialistes des réformateurs et des radicaux britanniques - les incitant à s'intéresser aux catégories des classes moyennes et populaires sous l'angle de leur participation à l'arène politique, et pas seulement comme objets du discours politique des élites ${ }^{9}$. En général donc, depuis les vingt dernières années, l'attention des historiens s'est beaucoup plus nettement tournée vers le loyalisme des années 1790, avec un intérêt non dénué d'empathie.

2 Il n'est donc pas surprenant que ceci ait débouché sur l'exploration de nombreux faits nouveaux sur la Grande-Bretagne pendant la décennie révolutionnaire, et ce dans 
différents domaines. On y reviendra ensuite, mais notons dès à présent qu'une attention nouvelle est par exemple portée à l'expérience écossaise pendant les années 1790. Jusqu'à très récemment, à quelques exceptions importantes près ${ }^{10}$, l'Écosse fut soit ignorée soit, au mieux, traitée comme un comté anglais de plus, dont on pouvait tirer quelques exemples afin d'étayer un argument. Pour analyser la réponse loyaliste à la Révolution française, la tendance a consisté à discuter le problème du citoyen actif : à la différence des années 1980, les historiens se sont en général davantage intéressés aux loyalistes situés en dehors de l'élite politique et sociale. Ces hommes et femmes ont été étudiés en tant que participants au processus politique, n'en déplaise à Edmund Burke. La place occupée par Burke lui-même dans le processus politique des années 1790, en dépit de recherches importantes sur sa vie et de la publication de ses discours et écrits, a été considérablement remise en question, et même diminuée. Enfin, les historiens se sont intéressés au paradoxe qui a vu l'État britannique autoriser, et même encourager, les classes moyennes et populaires à défendre une constitution élitiste. Kevin Gilmartin l'a bien formulé : «Comment l'opinion publique a-t-elle été mobilisée pour la défense d'un régime qui s'était engagé à limiter la force politique de l'opinion publique ? " ${ }^{11}$.

Malgré les interrogations récentes sur la centralité de Burke dans les débats britanniques des années 1790, cet article commence par passer en revue la littérature qui lui a été consacrée, puisque jusqu'à aujourd'hui il a été considéré comme un acteur incontournable. Les avancées historiographiques récentes seront ensuite abordées, à travers les débats portant sur les domaines suivants du loyalisme britannique pendant les années 1790 : l'idéologie, les organisations et les activités, les Églises, la presse et la propagande, enfin la question des masses conservatrices.

Edmund Burke

Pendant les deux dernières décennies, les études burkiennes ont connu deux avancées principales, suite à l'édition de la correspondance de Burke par T. W. Copeland entre 1958 et $1970{ }^{12}$. C'est d'abord le résultat d'une entreprise collective en plusieurs volumes, toujours en cours, éditée par Paul Langford: The Writings and Speeches of Edmund Burke ${ }^{13}$. Deux importantes biographies académiques ont également été consacrées au politicien irlandais.

Les volumes déjà parus des Writings and Speeches sont déjà des références. Les deux volumes qui nous intéressent ici, édités respectivement par L.G. Mitchell et R.B. McDowell, couvrent les écrits et les discours sur la Révolution française et les guerres ultérieures des années 1790-1794 et 1794-1797. Ils incluent aussi des introductions substantielles écrites par les éditeurs. L.G. Mitchell montre que Burke avait peu d'influence concrète sur la formulation de la politique du gouvernement britannique, tandis que R.B. McDowell suggère que la vision burkienne de la Révolution n'est qu'un révélateur de plus de son opposition générale à l'interférence de l'État dans la vie de ses sujets. F.P. Lock a mené ses recherches sur la façon dont Burke a conçu ses Réflexions sur la Révolution de France, et comment l'ouvrage fut reçu à l'époque, tandis que J.C.D. Clark a fourni une remarquable édition de ce célèbre ouvrage, dont l'introduction substantielle contextualise le livre par rapport à toute l'œuvre de Burke ${ }^{14}$.

6 The Great Melody: A Thematic Biography and Commented Anthology of Edmund Burke (Londres, 1992), de Conor Cruise O'Brien, est une longue mais stimulante analyse de la vie et du travail de Burke, qui prêche pour sa cohérence dans le temps. O'Brien examine tour à tour les activités et les écrits liés à l'Irlande, l'Amérique, l'Inde et la France. Il explique que les vues de Burke sur ces quatre problèmes sont toutes guidées par un 
leitmotiv, de même d'ailleurs que sa pensée sur la politique britannique, ce qui montre que, contrairement aux critiques que lui firent certains contemporains et certains historiens ${ }^{15}$, la position politique de Burke ne changea pas au cours du temps. Bien que les moyens adoptés aient pu évoluer (du soutien aux révolutionnaires américains à l'opposition aux insurgés français, par exemple), ses fins restèrent les mêmes (la défense d'une constitution britannique équilibrée). Le livre d'o'Brien est un travail personnel et attentionné, mais aussi une étude vivante avançant avec enthousiasme des opinions argumentées, dont les étudiants tireront un grand profit. La biographie de F.P. Lock ${ }^{16}$ est un travail académique, plus conventionnel et distancié, sans pour cela être fastidieux ou hostile : au contraire, le livre est très lisible, et il est probable qu'il deviendra l'ouvrage de référence sur le sujet. Le volume couvrant les années 1790 n'est pas encore publié, mais comme l'un des buts de F.P. Lock, en écrivant cette biographie, est d'en savoir plus sur l'auteur des Réflexions sur la Révolution en France, le premier volume, couvrant la vie de Burke jusqu'à 1784, est déjà utile pour ceux qui s'intéressent à sa carrière ultérieure. F.P. Lock a expliqué ailleurs que « les Reflexions ont toujours, et à raison, été considérées comme un produit de l'ensemble de la longue expérience politique de Burke ", car sa considération pour les formes constitutionnelles et les hiérarchies sociales de différents pays et continents ne fit que se développer et se renforcer au cours des décennies ${ }^{17}$. Les deux dernières décennies ont également vu un intérêt croissant, parmi les historiens du XVIII ${ }^{e}$ siècle, pour la satire politique, et ces travaux biographiques ont été complétés par Edmund Burke: A Life in Caricature (1996) de Nicholas K. Robinson, une magnifique collection de caricatures sur Burke publiées tout au long de sa carrière ${ }^{18}$.

7 Bien d'autres livres et articles ont été publiés sur la vision burkienne de la Révolution française. La plupart sont d'accord avec l'interprétation de O'Brien, selon laquelle l'opposition de Burke à la Révolution n'était pas contradictoire avec ses positions politiques antérieures sur d'autres sujets. Toutefois, des chercheurs comme John Whale et $\mathrm{F}$. P. Lock ont tenté de prendre du recul par rapport aux tentatives visant à retrouver une philosophie politique globale dans les Réflexions ${ }^{19}$, ouvrage qui doit au contraire être présenté comme le travail d'un politicien actif et pragmatique « dont l'écriture, [...] stratégique et provisoire plutôt que théorique et systématique ", doit être fermement inscrite dans son contexte historique ${ }^{20}$. D'après J.G.A. Pocock, la notion de contexte historique fournit une clef de lecture des Réflexions. Qu'il fulmine contre la manière dont était traitée Marie-Antoinette, ou qu'il se fraie un chemin à travers les documents juridiques et économiques sur la confiscation des terres de l'Église ou l'impression des assignats, Burke, selon John Pocock, s'est constamment insurgé contre le mépris des révolutionnaires pour leur contexte - c'est-à-dire contre le renversement complet de la société (la propriété, la présomption d'innocence, la prescription) ${ }^{21}$.

8 Enfin, les historiens se sont beaucoup intéressés récemment aux relations de Burke avec les émigrés français, ainsi qu'aux questions nationales. Colin Lucas expose en détail l'aide matérielle considérable qu'il a apporté aux émigrés, ce qui a été généralement entouré d'un voile pudique, tandis qu'Harvey Mitchell explique que Burke ne voulait aider que les plus purs des émigrés, ceux qui n'avaient absolument pas été contaminés par leur contact avec leur Révolution ${ }^{22}$. James $\mathrm{K}$. Chandler et Tom Furniss se sont penchés sur les idées de Burke sur la nation. Le premier de ces historiens a mis en valeur l'opinion de Burke selon laquelle la France avait perdu le droit d'auto-représentation, celui-ci étant incarné à l'Assemblée nationale par des « rustres ». Inversement, la constitution britannique, bien que moins représentative sur 
le plan numérique, était représentative, car elle correspondait parfaitement au caractère et aux intérêts du peuple anglais. Dans le même ordre d'idées, Burke présupposait qu'il s'adressait à des Anglais dans les Réflexions, et pourtant «savoir si et dans quel sens Burke écrivait en tant qu'Anglais est une question qui surgit dès le début de la lecture des Réflexions" ${ }^{23}$. T. Furniss nous rappelle que Burke fut préoccupé, tout au long de sa carrière politique, par la défense des identités locales contre la centralisation et la taxation métropolitaine. C'était le cas qu'il discute de l'Irlande, de l'Amérique ou de l'Inde ; concernant la Révolution française, il s'opposait également à ce qu'il appelait les «tendances centralisatrices et potentiellement totalitaires » des révolutionnaires ${ }^{24}$.

L'idéologie

9 Le débat britannique sur la Révolution française a longtemps été considéré comme l'apanage des seuls Burke et Paine. Cette vision s'est modifiée dans les deux dernières décennies. F.P. Lock a ainsi suggéré que Burke et Paine partageaient trop peu de terrain idéologique pour que l'on puisse parler d'une réelle discussion entre eux ${ }^{25}$. De même, comme l'a montré Mark Philp, la vague d'imprimés publiés à cette occasion ne peut pas vraiment être considérée comme un débat, étant donnée l'hétérogénéité des vues exprimées ${ }^{26}$. De leur côté, Gregory Claeys et Kevin Gilmartin sont allés jusqu’à remettre en cause non seulement la représentativité des positions de Burke, telles qu'exprimées dans les Réflexions, mais aussi sa centralité dans la controverse ${ }^{27}$. Ils affirment, comme l'ont fait avant eux H.T. Dickinson et J.G.A. Pocock, que la réponse conservatrice à la Révolution française était multiforme et qu'il est réducteur de n'y voir que des variations sur un thème initié par Burke ${ }^{28}$. K. Gilmartin a noté que Burke écrivit son pamphlet à destination des élites, sans jamais en faire un plan de mobilisation populaire ${ }^{29}$. G. Claeys a remarqué que la plupart des loyalistes étaient avant tout préoccupés par la défense de l'inégalité économique, s'opposant à ce qu'ils considéraient (à tort) comme la position de Paine sur le nivellement économique total. Ce même historien a également observé que la plupart des loyalistes n'étaient pas prêts à aller aussi loin que Burke sur nombre d'enjeux, comme l'idée que la classe politique britannique avait abandonné certains droits lors de la Glorieuse Révolution de $1688{ }^{30}$.

Il ne s'agit pas de tirer de ces quelques remarques l'idée que la force du conservatisme des années 1790 a été minimisée dans les années récentes. Dans une série de livres et d'articles publiés entre 1985 et 1995, H.T. Dickinson a analysé les idées conservatrices à la fin du XVIII ${ }^{e}$ siècle, développant une argumentation qu'il avait déjà exposée dans le dernier chapitre de son Liberty and Property (1977) ${ }^{31}$. En particulier, il insiste sur le fait que dans les années 1790, les radicaux ont perdu la bataille en Grande-Bretagne en raison de leurs faiblesses propres et de l'ampleur de la répression gouvernementale, point sur lequel la majorité des historiens s'accordaient déjà. Mais il démontrait que les conservateurs gagnèrent, au plan idéologique, par leur force de persuasion et leurs tactiques ${ }^{32}$. D'après cet historien, ce succès a été en grande partie dû à leur capacité à mobiliser des préjugés très répandus et des opinions déjà profondément ancrées dans les classes moyennes et populaires britanniques, en faveur des institutions traditionnelles et de l'ordre établi, tant au niveau de l'Église que de l'État. Ces groupes voyaient en effet dans la monarchie parlementaire, le règne de la loi et le maintien de l'ordre social un moyen de protéger leur liberté et leur prospérité. L'existence de cette croyance suscita une méfiance immédiate envers le radicalisme britannique et le jacobinisme français des années $1790{ }^{33}$. Le raisonnement de H.T. Dickinson renforce l'argumentation de Ian Christie dans ses Ford Lectures de 1983-1984, dans lesquelles il 
suggérait que la Grande-Bretagne avait évité une révolution dans les années 1790, malgré l'exemple français, en raison de la prospérité fondamentale de l'économie britannique, et parce que l'ordre social britannique favorisait le paternalisme et les mesures de bienfaisance envers les pauvres ${ }^{34}$.

D'autres chercheurs ont confirmé cette interprétation (bien évidemment dans d'autres buts), au point qu'en 1991 John Dinwiddy forgea l'expression de «consensus dickinsonien " ${ }^{35}$. L'article de Linda Colley, "The Apotheosis of George III », avait déjà montré l'attraction et la popularité de la monarchie dans les années 1790. L'ouvrage de Marilyn Morris, The British Monarchy and the French Revolution, a poursuivi le débat sur la monarchie britannique pendant la même décennie, expliquant que la Révolution française avait obligé les loyalistes aussi bien que les radicaux à modifier leurs idées sur leur propre monarchie. Tandis que les radicaux préféraient en général une réforme interne de la monarchie à un système républicain, les loyalistes avaient tendance à incorporer les vertus républicaines à leur représentation patriarcale de la monarchie ${ }^{36}$. Dans son étude de la pensée conservatrice anglaise dans les années 1790, Philip Schofield écrit qu'» il se peut que l'attraction exercée par l'idéologie conservatrice n'ait résidé que dans sa défense des intérêts en place, mais les certitudes ajoutées à l'intérêt engendrèrent un adversaire bien plus acharné de l'agitation radicale " ${ }^{37}$. Le livre de J.C.D. Clark, English Society, 1688-1832, souligne la continuité de l'influence politique et sociale de la monarchie, de l'aristocratie et de l'Église d'Angleterre au tournant du XIX ${ }^{\mathrm{e}}$ siècle. Cet historien y explique qu'à l'échelle mondiale, la vision des conservateurs mettant l'accent sur l'ordre et l'obéissance était encore suffisamment puissante pour ne pas être réellement menacée par le nombre relativement faible des radicaux politiques et religieux de la fin du XVIII ${ }^{e}$ siècle. On pourrait aller jusqu'à avancer que le XVIII ${ }^{e}$ siècle a survécu en Grande-Bretagne jusqu'au milieu du siècle suivant ${ }^{38}$. Stella Cottrell et Michael Duffy ont montré qu'une gallophobie virulente était un autre préjugé profondément ancré dans le peuple britannique, qui fut exploité avec succès par les loyalistes afin de gagner cette lutte ${ }^{39}$. Toutefois, John Dinwiddy a contesté la force de l'argumentation conservatrice, suggérant que, loin de constituer un atout, le réemploi d'idées et d'arguments familiers était en fait un signe du manque d'inspiration et de la paresse des propagandistes conservateurs. De plus, les phases de soutien puis de mécontentement envers le gouvernement disparurent, puis réapparurent, à maintes reprises pendant le long conflit militaire avec la France, davantage en fonction des circonstances qu'en raison de la qualité de l'argumentation conservatrice ${ }^{40}$.

12 Enfin, la manière dont les conservateurs-loyalistes écossais ont réagi à la Révolution française a commencé à susciter des travaux depuis quelques années. Les écrits les plus récents sur le loyalisme britannique, comme ceux de J. Cookson et A. Gee ${ }^{41}$, ont pris soin d'intégrer la dimension écossaise, tandis que les travaux centrés sur le loyalisme écossais ont pris la suite de ceux portant sur le radicalisme, à l'instar de l'évolution observée pour le loyalisme anglais ${ }^{42}$. Des travaux portant à la fois sur le loyalisme et le radicalisme en Écosse s'inscrivent dans la voie ouverte par Henry Meikle dans son livre séminal de 1912, Scotland and the French Revolution. Cependant, si un nombre encore réduit mais significatif de travaux portant sur le radicalisme se sont peu à peu accumulés dans les années 1980 et au début des années 1990, il a fallu plus longtemps pour que les conservateurs suscitent un intérêt comparable ${ }^{43}$, et peu de choses ont été pour l'instant publiées sur ce sujet. La thèse de doctorat d'Atle Wold, The Scottish Government and the French Threat, 1792-1802 (2003), est la contribution la plus substantielle jusqu'à présent, consacrant deux chapitres aux activités loyalistes et à 
leur idéologie ${ }^{44}$. Il y a montré que les arguments avancés par la propagande conservatrice en Écosse étaient généralement identiques à ceux exprimés dans la propagande loyaliste anglaise, à l'exception du raisonnement que l'on trouve parfois sur les bénéfices de l'union britannique pour les Écossais. Les Cheap Repository Tracts d'Hannah More furent ainsi révisés pour correspondre à un cadre écossais et «traduits » dans un dialecte écossais peu après leur publication en Angleterre ${ }^{45}$.

Les organisations et leurs activités

13 Les travaux portant sur les groupes conservateurs et leurs activités ont suivi trois directions principales. Les ouvrages se sont d'abord concentrés sur la manière dont le peuple s'est rallié à la contre-révolution, soulignant le paradoxe d'une mobilisation qui coexistait avec l'idéologie d'une représentation de la propriété. Second chantier, le débat pour savoir jusqu'à quel point les Volontaires étaient loyalistes, par opposition à une organisation principalement tournée vers la défense militaire. Enfin, dernière direction, on trouve les nombreux travaux sur le loyalisme écossais.

14 La production historiographique ancienne sur les associations loyalistes (comme l'Association pour la préservation de la liberté contre les républicains et niveleurs, ciaprès APLP) avait discuté de l'étendue, de la composition et de la nature de ces groupes de citoyens. D'après cette interprétation, les loyalistes adoptèrent une vision conservatrice de la menace révolutionnaire pour l'État britannique et la constitution, et se groupèrent pour faire circuler des pamphlets loyalistes et casser le radicalisme local, ou pour d'autres, par simple opportunisme ${ }^{46}$. En 1983, dans son For King, Constitution and Country: The English Loyalists and the French Revolution, Robert R. Dozier examinait en détail les associations loyalistes ainsi que les mouvements de Volontaires ultérieurs. Il y montrait que la force de la ferveur loyaliste et la popularité de l'activité loyaliste avaient tendance à croître ou diminuer en fonction des événements en France, et de la menace militaire française, réelle ou perçue. Le même livre contenait des développements précis sur les protestations politiques intérieures et le rôle des loyalistes dans leur suppression. Il est frappant de voir que R. Dozier avait déjà soulevé le problème auquel était confronté l'État pour mener une guerre des idées de grande ampleur : la nécessité d'impliquer et même d'inviter les sujets ordinaires à participer à la suppression de l'opposition intérieure, et à défendre une constitution non représentative qui privilégiait la propriété et la naissance ${ }^{47}$.

Harry Dickinson ne s'est pas borné à l'examen de l'idéologie des loyalistes britanniques dans les années 1790 , mais a également exploré leurs activités et leurs tactiques ${ }^{48}$. Il a notamment mis au jour l'existence de segments de la société britannique profondément politisés, bien que n'appartenant pas à l'élite. En plus de l'éventail des activités politiques concernées qui était considérable, l'auteur a su mettre en évidence une volonté largement partagée d'accepter et de se conformer aux directives politiques de la propagande loyaliste. Il a aussi souligné que les conservateurs adoptèrent nombre des tactiques des radicaux et des politiciens réformateurs afin de rallier le support populaire, comme des campagnes de pétitions, des manifestations de masse, ou encore des clubs et associations. D'après lui, les efforts des loyalistes furent bien plus fructueux que ceux des radicaux, amenant des contingents importants à manifester un soutien à leur cause. Il affirme en outre que les loyalistes gagnèrent leur bataille d'organisation, aussi bien que leurs disputes idéologiques, avec les radicaux. Il apparaît de plus que si le conservatisme populaire et le loyalisme militant furent souvent encouragés par les élites, il serait trompeur de considérer que ces forces étaient totalement sous le 
contrôle de ces dernières. Le nombre même des militants qui furent amenés à exprimer un support à la cause loyaliste suggère en effet fortement l'impossibilité d'une contrainte ou d'une manipulation systématique. Alan Booth est arrivé aux mêmes conclusions: «Il est clair que les foules soutenant l'Église et le Roi étaient tout simplement trop nombreuses pour que la théorie des mercenaires fournisse un modèle explicatif adéquat» ${ }^{49}$. H.T. Dickinson en conclut qu'en se réjouissant des manifestations populaires soutenant le système politique en place, l'élite gouvernementale «se devait d'accepter que les préjugés populaires échappent de temps en temps à son contrôle ${ }^{50}$.

David Eastwood a centré son attention sur le dilemme devant lequel se trouvait l'élite gouvernementale: le désir de mobiliser le soutien public afin d'assurer la stabilité intérieure pendant la guerre, tout en refusant d'encourager une participation populaire permanente au processus politique ${ }^{51}$. Le mouvement dit "loyaliste" fournit un exemple révélateur de l'extrême difficulté pour les membres du gouvernement de promouvoir ce soutien public sans pour autant cesser de le contrôler. D. Eastwood a ainsi remarqué que les chefs d'accusation retenus par le Grand Jury du Middlesex en 1792, par les présidents de la cour d'appel (chief justices) Ashurst et Mainwaring, furent déterminants dans la formation de l'association, même si les autorités locales y eurent également un rôle crucial ${ }^{52}$. Dans un article de 1996, Michael Duffy a apporté des éléments nouveaux sur les origines de l'association, démontrant de façon probante que ce mouvement trouvait ses origines à la fois dans l'action du gouvernement et à l'extérieur de celui-ci. C'est en dehors du gouvernement que cette organisation fut d'abord formée, le projet initial, publié le 23 novembre 1792, était l'œuvre de John Reeves, d'abord à l'insu des ministres. Ces derniers se saisirent ensuite des propositions de Reeves et les modifièrent à leur avantage, avant que Reeves ne les publie à nouveau le 26 novembre ${ }^{53}$. Répondant à la mise en garde de Mark Philp, selon lequel tout élargissement de la participation politique dans les années 1790 posait problème à l'élite conservatrice, Kevin Gilmartin a quant à lui soutenu que l'élite gouvernementale est toujours restée consciente du défi que représentait son soutien au mouvement de l'Association Loyaliste, à laquelle il ne fallait surtout pas laisser une marge de manœuvre infinie:

«La gestion des conséquences involontaires, loin d'apparaître après coup ou de façon larvée, fut au contraire une dimension constitutive du projet conservateur, que l'on observe déjà dans les premières tentatives de mettre en place une réponse populaire à la Révolution française. ${ }^{54}$

17 L'imitation des tactiques radicales par les conservateurs, qu'a observée H. T. Dickinson, était nécessaire pour empêcher le radicalisme d'occuper cet espace politique. Si cet appel au soutien public pour obtenir l'obéissance politique peut sembler paradoxal, il était pourtant délibéré :

«L'Association s'efforça de formaliser et de contrôler la manière dont l'argumentation sur la satisfaction du peuple, à destination des lecteurs ordinaires, pénétra le discours public [...] [et] l'arène au sein de laquelle il serait permis aux "segments ouvriers du public britannique" de parvenir à une prise de conscience politique. ${ }^{55}$

Une version encore plus marquée de cette difficulté survint avec l'émergence du Corps des Volontaires. Comme l'a remarqué Austin Gee, les récents précédents américains, irlandais et français d'une participation militaire citoyenne n'étaient guère encourageants pour l'élite britannique ${ }^{56}$. Ajoutons que la majorité de la population ne 
soutenait pas forcément cette mobilisation, comme le prouvèrent les émeutes contre le recrutement, ainsi que les protestations populaires contre les impôts de guerre élevés 57. Dans un article publié en 1956, J.R. Western avait montré que l'organisation d'une défense militaire avait un rôle politique autant que militaire: elle était conçue pour s'intégrer à la stratégie loyaliste contre le radicalisme intérieur. Cette interprétation a été soutenue par des chercheurs comme R. Dozier et H. T. Dickinson ${ }^{58}$. Plus récemment, David Eastwood et John Cookson ont insisté sur l'autonomie de nombre de ces unités de Volontaires, ainsi que sur leurs préoccupations d'abord locales ${ }^{59}$. D'après D. Eastwood, ce localisme était à la fois la force principale et la faiblesse majeure de ce mouvement. Ce sont souvent des considérations locales qui furent l'élément déclencheur dans la fondation d'un corps, et leur financement initial était souvent le fruit de souscriptions locales. Toutefois, cet ancrage local avait souvent pour corollaire le refus de servir hors de leur comté. En outre, trouver des sources de financement constantes était généralement difficile, une fois épuisées les réserves d'argent. D. Eastwood suggère néanmoins que "ce type de patriotisme opérait au niveau subnational, ce qui s'accordait parfaitement avec les traditions décentralisatrices de l'État anglais " ${ }^{60}$. J. Cookson a lui aussi contesté l'idée que la loyauté des Volontaires envers l'État résultait de leurs appartenances locales, en minimisant l'importance de leur rôle dans la suppression du radicalisme intérieur. D'après lui, la principale fonction des Volontaires fut d'être un rempart contre l'invasion française, ce qui contribua dans le même temps à établir un "patriotisme de la défense nationale " capable d'unir des individus aux opinions politiques variables - ce que le loyalisme contre-révolutionnaire ne pouvait faire. Linda Colley a également soutenu que la principale raison de s'engager était le désir de défendre sa maison et son foyer contre une hypothétique invasion française ${ }^{61}$.

19 Cet argument est pour l'essentiel valable, et il semble indéniable que nombreux furent les Volontaires, pris individuellement, à ne pas être très motivés par leur hostilité idéologique à la Révolution française. Certains d'entre eux, comme l'ont montré J. Cookson et L. Colley, profitèrent de leur adhésion à un corps pour promouvoir leur statut personnel au sein de la communauté locale, tout particulièrement avant les incitations à l'engagement en masse en 1798. De plus, le fait de privilégier les enjeux locaux et personnels sur les questions nationales renvoie à la démonstration plus générale que fit Paul Langford en $1991{ }^{62}$. Plus récemment encore, A. Gee, qui a décrit et analysé de la manière la plus complète à ce jour le mouvement des Volontaires britanniques, a exprimé à son tour son désaccord avec l'interprétation de J. Western, préférant à l'idée d'un loyalisme militant des Volontaires leur défense militaire de l'État britannique ${ }^{63}$. Selon A. Gee, les Volontaires furent en général loyaux envers la couronne, et leur rôle fut d'abord d'être des agents de l'ordre. Seule une minorité se perçut comme une force activement engagée politiquement, anti-radicale et contrerévolutionnaire ${ }^{64}$.

20 Toutefois, il n'en reste pas moins que le mouvement des Volontaires lui-même était assimilé à la défense de la constitution britannique, même si son origine résidait dans le désir de renforcer les défenses militaires du pays, et même si à l'échelle individuelle les Volontaires ne s'impliquaient pas souvent dans la cause anti-radicale. Les idées loyalistes se diffusèrent dans la mise en place des constitutions, dans la propagande et même dans les noms des compagnies de Volontaires, tels les Loyal Warrington Volunteers ${ }^{65}$. De plus, il est probable que certains Volontaires aient été conscients d'être guidés sur 
le chemin du loyalisme. Les membres des Volontaires d'Edinburgh, du moins dans les premiers temps de l'existence de ce corps en juin 1794, devaient signer une déclaration incluant les mots suivants :

«Nous réprouvons la doctrine du suffrage universel, et les principes politiques jacobins ou français. Nous désapprouvons toutes ces sociétés qui ont été formées, et ces réunions qui ont été tenues en Grande-Bretagne, ces deux dernières années, sous le titre de Friends of the People, British Convention, et appellations similaires; et nous nous efforcerons d'empêcher, par tous les moyens légaux à notre disposition, la formation de sociétés de ce type, et les réunions de ce type, et de faire tout notre possible pour neutraliser les efforts de ceux qui, soit de façon avouée, soit sous le prétexte de la réforme parlementaire, s'évertuent à renverser la constitution britannique. ${ }^{66}$

Une fois devenus Royal Edinburgh Volunteers, les membres écoutaient, à chaque jour de l'an, un sermon aux idées souvent ouvertement loyalistes et anti-radicales. En 1795, leur chapelain, le doyen George Baird de l'Université d'Edinburgh,

« fit des observations sur la constitution française, et discourut en termes très vifs sur la misère de cette malheureuse nation. Il souligna le contraste qui existait avec l'heureuse constitution qui nous apportait tant de bienfaits, et pour la défense de laquelle les Royal Edinburgh Volunteers s'étaient élevés avec tant d'empressement.» ${ }^{67}$

En 1798, il prêcha d'après le Deutéronome $23: 9$ : "Quand tu iras camper contre tes ennemis, tu te garderas de tout mal ${ }^{68}$. Il se peut toutefois que les troupes d'Edinburgh aient été originales dans cet étalage de positions ouvertement loyalistes. D'après A. Gee, «les Volontaires étaient davantage une force constitutionnelle que partisane, soutenant le système existant plutôt que l'administration alors en place » ${ }^{69}$.

Touchant l'activité loyaliste en Écosse, la thèse de doctorat de John Brims (1983) sur le radicalisme écossais a évidemment pris en compte les loyalistes ${ }^{70}$. Les centres d'intérêt de la thèse que David Brown (1989) a consacrée à « Henry Dundas et le gouvernement de l'Écosse " étaient suffisamment variés pour offrir également des études prospectives sur les loyalistes ${ }^{71}$. La thèse d'Atle Wold (2003) contenait quant à elle un chapitre discutant des activités loyalistes ${ }^{72}$, dans lequel il concluait que les associations loyalistes n'étaient pas aussi puissantes en Écosse qu'en Angleterre. Il ajoutait que bien d'autres formes de loyalisme furent plus vigoureuses, ou relativement mieux représentées en Écosse que plus au sud, comme les résolutions, les conseils aux membres du gouvernement, le service militaire personnalisé (y compris les Volontaires), et des contributions financières. Mon article «A City Invincible? Edinburgh and the War against Revolutionary France " renforce les conclusions de D. Eastwood, J. Cookson et A. Gee en montrant que le loyalisme émergeait souvent de considérations locales bien plus que nationales. Dans sa discussion des Volontaires des Highlands, Andrew Mackillop arrive aux mêmes conclusions ${ }^{73}$. Dans un ouvrage à paraître intitulé Scotland and the French Revolution, sous la direction de Bob Harris ${ }^{74}$, A. Wold a consacré un chapitre aux «Scottish Attitudes to Military Mobilization and War in the 1790 s ». Cependant, l'absence de toute étude locale ou même régionale sur le loyalisme écossais, appuyé sur une recherche dans les archives locales, sans même parler d'une synthèse, reste frappante.

Les Églises

Les Églises de la fin du XVIII ${ }^{e}$ siècle ont suscité un intérêt constant au cours des deux dernières décennies, notamment en ce qui concerne leurs idées politiques. La thèse de doctorat de Nancy Murray (1975) avait déjà prouvé la place prééminente du clergé dans 
la guerre de propagande pendant la Révolution française. Elle y analysait minutieusement les opinions exprimées sur la Révolution par différentes confessions et partis de l'Église d'Angleterre, et l'impact de la décennie révolutionnaire sur leurs différentes destinées. Y était notamment montré qu'en s'opposant ardemment à la Révolution française et au radicalisme intérieur, ainsi qu'en soutenant de façon tout aussi déterminée le gouvernement et la guerre contre la France, le clergé orthodoxe de l'Église d'Angleterre consolida la place de l'Église comme auxiliaire privilégié d'un État séculier, tout en diminuant la probabilité à court terme d'un retrait des Test and Corporation Acts ${ }^{75}$. Elle y expliquait enfin que les Évangélistes, tant les membres de l'Église anglicane que les Dissenters, s'opposèrent aussi à la Révolution française et soutinrent la guerre.

Deryck Lovegrove a confirmé cette vision des dissidents évangéliques, mais a montré qu'ils cherchèrent, quand c'était possible, à éviter les discussions politiques, essayant ainsi de prouver leur fiabilité politique ${ }^{76}$. D'après le travail de David Hempton, les méthodistes (qui n'étaient pas, bien évidemment, toujours dissidents avant 1795), étaient souvent plus occupés à régler leurs disputes internes qu'à prêcher des sermons politiques ${ }^{77}$. Dans un article de 1982, William Stafford a noté que si les dissidents évangélistes étaient généralement loyalistes, ils avaient également tendance à blâmer les péchés de leur pays et n'avaient de cesse de réclamer sa réformation morale : «Leur sens profond du péché travaillait dans le sens de l'obéissance, mais contre le nationalisme ${ }^{78}$.

Robert Hole s'est intéressé aux sermons politiques et aux pamphlets de propagande conservatrice écrits par les membres du clergé dans les années $1790{ }^{79}$. Même si beaucoup d'hommes d'Église utilisaient des logiques argumentatives contrerévolutionnaires, ces textes n'étaient pas de simples applications de la pensée de Burke à leur situation particulière - à l'instar des pamphlets loyalistes écrits par les journalistes séculiers. Au contraire, c'est en termes de théologie politique qu'ils s'exprimaient, liant les événements en France et ailleurs aux preuves scripturaires et aux débats sur les relations entre l'Église et l'État. Les devoirs des sujets étaient un autre thème fréquent, autour des questions de hiérarchie, d'ordre et d'obéissance. Dans tous les cas, il fallut deux ou trois ans avant que ces sermons politiques conservateurs, comme tous les autres écrits conservateurs, n'en viennent à suivre les positions exprimées par Burke dans ses Réflexions, à la fin $1790{ }^{80}$.

Sur l'Écosse, l'étude majeure de Richard Sher (1985) concerne les leaders du parti Moderate (non-évangélique) de l'Église écossaise. Il y explique que l'hostilité des prêcheurs modérés envers la Révolution française était bien plus franche que leur opposition à la Révolution américaine ${ }^{81}$. Il y montre que c'est en référence au Covenant qu'ils exprimaient généralement leur opposition à la Révolution française. Les lecteurs et les auditeurs de leurs sermons se voyaient ainsi rappeler la souveraineté de Dieu et son intervention fréquente dans les affaires de ce monde, la punition que subirent les nations qui s'étaient rebellées contre lui, et la nécessité d'une repentance nationale et d'une réformation morale, en échange desquelles Dieu avait promis de suspendre son courroux et d'interrompre les calamités qu'il avait envoyées. De tels discours pouvaient aussi s'appliquer à la Grande-Bretagne, dont les sujets étaient coupables d'ingratitude envers Dieu, qui leur avait fait grâce de la constitution britannique. Les radicaux et réformateurs étaient tout particulièrement visés, comme l'étaient aussi ceux qui avaient "abusé » des privilèges religieux de cette constitution: les athées, les 
schismatiques, et tous ceux qui avaient succombé aux péchés du profit et de la cupidité commerçante. Pour ces raisons, les Britanniques devaient s'unir sans réserve dans le soutien à la guerre contre la France, et respecter la constitution britannique et la «vraie religion» ${ }^{82}$. À propos des Évangélistes, ou parti «populaire » de l'Église d'Écosse, John McIntosh a démontré qu'ils étaient eux aussi très hostiles à la Révolution française. En effet, s'ils n'étaient pas forcément très éloignés des doctrines enseignées par les prêcheurs modérés sur le sujet, leur angle d'approche était le péché originel et la perversion universelle. Pour contenir l'inclination naturelle des hommes vers le mal, le gouvernement civil était donc une nécessité, et le gouvernement une institution divine, contre laquelle la rébellion était un péché ${ }^{83}$. Ce soutien au gouvernement et à la constitution n'était pas inconditionnel: parmi les Évangélistes, certains reconnaissaient le besoin d'une réforme politique modérée, qui devrait néanmoins être reportée à un temps plus opportun ${ }^{84}$. De même que les schismatiques, les dissidents écossais s'opposèrent généralement à la Révolution française, particulièrement à la persécution des chrétiens. C'est aussi dans leurs rangs que l'on avait le plus de chances de trouver les sympathisants de la Révolution et de la réforme politique en GrandeBretagne ${ }^{85}$.

La presse et la propagande

Dès 1976, Gayle Trusdel Pendleton rejetait l'image classique de la presse loyaliste des années 1790, décrite comme "Burke et une poignée de partisans incompétents, aboyant de manière stérile contre le radicalisme et la révolution ». D'après l'auteure, une description plus juste des publicistes conservateurs pendant cette décennie en faisait «ceux qui étaient des compagnons de Burke à proprement parler, et non ses disciples dans cette cause ${ }^{86}$. Les publications récentes sur la presse conservatrice des années 1790 ont renforcé cette affirmation, en l'étayant de sources bien plus fournies. Une autre tendance s'est développée dans la foulée d'un article écrit par Herbert M. Atherton en 1982, pour étudier la satire graphique. C'est plus généralement pour l'ensemble des dix-huitièmistes que ce domaine d'exploration a pris son essor, à la suite des travaux de Mary Dorothy George au milieu du XXe siècle ${ }^{87}$.

D'après l'expression de H. Dickinson, la presse loyaliste des années 1790 a déversé « un véritable déluge de propagande conservatrice et loyaliste ", et les spécialistes de cette presse ont commencé à en explorer les diverses ramifications ${ }^{88}$. Philip Schofield s'est intéressé à la pensée conservatrice sur la Révolution, la guerre et la constitution britannique, se focalisant sur des pamphlets politiques relativement denses. Il a montré que le débat autour de la Révolution dominait la politique nationale et extérieure britannique pendant toutes les années 1790 et que les conservateurs, du moins dans la première partie de la décennie, s'accordent pour tout subordonner à la défense de la constitution et au soutien à la guerre contre la France ${ }^{89}$. Emily Lorraine de Montluzin a fait l'analyse des contributeurs de l'Anti-Jacobin Review, revue mensuelle de premier plan dans la diffusion des idées contre-révolutionnaires, entre 1798 et $1800{ }^{90}$. Édité par John Gifford, le périodique employait des journalistes très compétents, comme John Reeves, John Bowles, William Jones, John Taylor et William Cobbett. Toutefois, si 20 des 70 contributeurs de la revue, lors des deux premières années de son existence, furent des journalistes ou des écrivains professionnels, 15 étaient des maîtres d'école et des universitaires, tandis que les autres étaient un mélange d'hommes politiques, d'hommes de loi, de médecins et de petits commerçants. Environ un tiers habitait à Londres, un second tiers, appartenant principalement au clergé, était dispersé dans toute l'Angleterre, tandis que les autres, lorsque leur lieu de résidence a été identifié, 
habitaient en Écosse et en Irlande. Enfin, cinq étaient des émigrés français résidant en Angleterre ${ }^{91}$.

Robert Hole s'est intéressé à la propagande destinée au peuple ${ }^{92}$. À l'instar de G. Trusdel Pendleton, il tirait la conclusion que ces journalistes conservateurs n'étaient pas seulement des imitateurs serviles d'un Burke adapté aux masses :

«En général, l'influence de Burke sur les pamphlets ne s'est pas tant fait sentir dans les arguments que dans la manière de penser. Dans une minorité de libelles destinés au segment le plus "cultivé" du marché populaire l'atmosphère et le ton burkiens dominent, et l'on peut retrouver ses arguments précis. [...] Mais ceux-ci [...] ne sont qu'en partie représentatifs. ${ }^{93}$

L'étude du roman anti-jacobin qu'a menée Matthew Grenby débouche sur des conclusions similaires: cette source souvent négligée est pourtant très utile pour comprendre la réponse des conservateurs britanniques à la Révolution française. Ces publications, qui n'avaient aucune prétention littéraire, trouvent leur origine dans la tradition du roman populaire, qui fut longtemps ignoré par la critique littéraire car il était d'abord destiné à un lectorat de jeunes femmes et aux classes populaires. Pendant la décennie révolutionnaire, ces œuvres acquirent un certain degré de légitimité littéraire, car les auteurs, les éditeurs comme les critiques comprirent leur potentiel pour véhiculer les valeurs conservatrices dans un public assez vaste ${ }^{94}$.

Jeremy Black, Bob Harris et Hannah Barker ont prolongé l'enquête sur la presse en s'intéressant à celle de Londres mais aussi aux journaux provinciaux, pour voir comment la Révolution était perçue dans ce média influent ${ }^{95}$. H. Barker a noté que la presse était dans une situation privilégiée pour profiter de l'appétit vorace du public britannique pour les informations sur la Révolution française, ce qui explique que «sa version des événements était extrêmement influente " ${ }^{96}$. Le travail de B. Harris a mis fin à la pénurie de publications sur la presse politique écossaise, qui touchait l'ensemble du XVIII ${ }^{e}$ siècle ${ }^{97}$. Les positions de la presse loyaliste écossaise reflétaient la tendance générale des conservateurs britanniques, qui s'opposèrent à la Révolution à l'automne 1792, et l'on observe bien d'autres similitudes avec la presse anglaise pendant cette décennie. Ces journaux ne tenaient aucun compte du radicalisme intérieur, sauf pour annoncer sa défaite, et ménageaient dans leurs colonnes un espace substantiel pour imprimer les proclamations conservatrices et les adresses des sociétés commerciales ainsi que les rapports sur les activités loyalistes. La presse conservatrice soutint la guerre contre la France révolutionnaire, faisant mousser les victoires et minimisant les défaites. En Écosse, plus nettement encore qu'en Angleterre, la presse radicale était vaincue avant 1794, la résistance au gouvernement n'étant plus représentée que par l'opposition libérale Whig, comme le Scots Chronicle qui émergea en 1796. L'ampleur de la Révolution, comme événement politique, encouragea également la presse écossaise à s'engager dans des avancées techniques variées, comme un usage plus fréquent des titres, des éditoriaux politiques, ou encore une typographie spécifique pour la relation des « atrocités " ${ }^{98}$.

33 En ce qui concerne les dessins satiriques, les volumes coordonnés par Michael Duffy et Harry Dickinson, au sein d'une collection consacrée aux caricatures du XVIII e siècle, se situent dans la même perspective ${ }^{99}$. H. Dickinson a reproduit nombre de caricatures défendant la constitution, mais aussi beaucoup qui la critiquent. D'après lui, il était bien plus difficile pour les artistes satiriques de dépeindre les vertus de la constitution britannique que de montrer par l'image les défauts de leurs opposants radicaux et réformistes et le danger de leurs projets de changements politiques. M. Duffy a quant à 
lui expliqué que ces gravures donnaient une vision déformée jusqu'à l'absurde des événements français - la Révolution tombant rapidement sous la coupe des masses de non-possédants - mais cette vision fut néanmoins « largement diffusée et extrêmement fructueuse ${ }^{100}$.

L'impact de la Révolution française sur la gravure a fait l'objet des travaux de Diana Donald et David Bindman. Selon D. Donald, la satire visuelle montre que le loyalisme a réutilisé, à ses propres fins, des armes forgées par les réformateurs. Tandis que les éditeurs et les vendeurs d'images favorables aux réformes étaient pourchassés et harcelés par l'APLP, les loyalistes continuaient à commander des gravures satiriques pour défendre leur propre cause. Ces dernières recouraient à la gallophobie traditionnelle en Angleterre, montrant Louis XVI guillotiné, et opposant l'état de dévastation de la France républicaine au bonheur régnant en Grande-Bretagne. D’après la même historienne d'art, il était souvent plus facile, dans ces gravures, de s'en prendre aux ennemis jacobins plutôt que de décrire les bénédictions dont jouissait la Grande-Bretagne. Ainsi, le bonnet de la liberté qu'arborait traditionnellement Britannia pouvait facilement être confondu avec les nouveaux symboles révolutionnaires français, ce qui explique sa disparition progressive dans l'attirail de Britannia, à partir du milieu des années 1790. D. Bindman explique quant à lui qu'à la fin 1793, la plupart des artistes et des éditeurs satiriques ne produisaient plus que des gravures probritanniques et loyalistes - tout en gardant le droit de critiquer Pitt pour sa politique intérieure et de se moquer du roi ${ }^{101}$. Napoleon and the Invasion of Britain, un ouvrage édité par Alexandra Franklin et Mark Philp, reproduit 92 images et textes dont beaucoup sont imprégnés de gallophobie mais aussi de soutien envers le gouvernement britannique. Cette tendance se maintient jusqu'à la disparition de la menace d'invasion, lorsque la satire du gouvernement britannique devient à nouveau populaire ${ }^{102}$. Le livre oppose deux périodes, montrant l'état de division de la société britannique lorsqu'elle fit face à la menace d'invasion française en 1798 , et son unité contre la menace napoléonienne entre 1803 et 1805.

S'intéressant à la consommation de masse des estampes satiriques, D. Donald a noté que certaines gravures étaient spécialement adaptées pour la décoration d'objets en céramique. Ceci est un indicateur solide de leur popularité, alors que les subventions (dont ils bénéficiaient) et la distribution gratuite d'autres gravures laissent planer un doute sur leur impact réel. Eirwen C. Nicholson a toutefois développé l'idée que les images satiriques touchaient un nombre de gens bien inférieur à ce que l'on a généralement supposé. En effet, les gravures loyalistes, comme les journaux, ne prêtaient guère d'attention aux sociétés radicales britanniques, choisissant plutôt Charles James Fox et ses acolytes pour incarner la menace jacobine. Ceci semble suggérer que ces images étaient principalement vendues aux parlementaires et à l'élite gouvernante ${ }^{103}$. Bien que le livre de Timothy Clayton donne aux estampes une place centrale dans la culture nationale du XVIII ${ }^{\mathrm{e}}$ siècle, il ne s'agit ici que d'une culture d'élite. L'exemple de Gillray est révélateur : incité à vendre des gravures de sa série sur les Consequences of a Successful French Invasion (1798) au prix de six pence l'une afin d'en augmenter les ventes, il se rendit compte que «les acheteurs intéressés les auraient sans barguigner payées une demie couronne ${ }^{104}$.

Enfin, trois livres portant sur des artistes spécifiques, et deux sur les représentations satiriques d'individus précis, ont permis d'étoffer notre compréhension et de nourrir nos connaissances dans ce domaine. Ce sont les travaux de Robert Patten sur George 
Cruikshank, David Alexander sur Richard Newton, et Richard Godfrey sur James Gillray dans la première catégorie, et ceux de Nicholas Robinson sur les caricatures d'Edmund Burke et de Vincent Carretta sur George III dans la seconde catégorie ${ }^{105}$. S'il est probable que Gillray ait été payé par le gouvernement pour réaliser certaines de ses gravures anti-révolutionnaires, son travail ne fut pas constamment au service de la propagande loyaliste. Ajoutons qu'aucun des ouvrages mentionnés n'est centré sur les gravures conservatrices.

Le conservatisme des masses?

La question fondamentale, que formulait Mark Philp en 1995, reste pourtant d'évaluer la portée de cette propagande conservatrice, à la fois sur les idées et sur les actions de ceux qui y succombaient. En d'autres termes, en dehors des activistes patentés, jusqu'à quel point les Britanniques ordinaires étaient-ils loyalistes ? Furent-ils convaincus par la propagande, qu'ils la lisent eux-mêmes ou qu'elle leur soit lue ? Ou bien le rôle de celle-ci ne se limitait-il, comme l'affirme M. Philp, qu'au divertissement ${ }^{106}$ ? Comme d'autres avant lui, cet historien a en effet suggéré que bien d'autres motivations ont pu se combiner avec une loyauté authentique : au premier chef le désir d'acquérir un statut social et politique, en s'engageant dans une association loyaliste ou dans un autre type d'activité loyaliste ${ }^{107}$. Il a aussi expliqué qu'à elle seule, la force des arguments conservateurs ne pouvait suffire à persuader les masses d'arrêter de prendre part aux manifestations et aux campagnes radicales. Il serait donc imprudent d'insister avec trop d'ardeur sur la «loyauté naturelle» du peuple britannique. Le gouvernement britannique, et son premier ministre, ne se sont peut-être pas rendus coupables d'un "règne de la Terreur " ${ }^{108}$, mais les politiques répressives ont peut-être suffi à défaire les radicaux et à calmer le reste de la population ${ }^{109}$.

Comme on l'a vu, Ian Christie a expliqué qu'associées à la propagande conservatrice, la puissance relative de l'économie britannique et une dose minimale de paternalisme des élites envers les pauvres permettent d'expliquer l'absence d'une réelle menace révolutionnaire en Grande-Bretagne pendant cette période ${ }^{110}$. Roger Wells a rejeté cette analyse, amassant quantité de preuves montrant les sévères problèmes économiques rencontrés par nombre de Britanniques pendant les années 1790 et au début des années 1800 . Ceci confortait donc l'hypothèse plus ancienne formulée par E.P. Thompson, selon laquelle le gouvernement britannique fut bien chanceux d'avoir pu éviter une insurrection dans les années 1790, au regard de la situation désespérée d'une grande partie de la population. $R$. Wells quant à lui estimait avoir réduit à néant l'idée d'une vague notable de loyalisme populaire, en prouvant que l'opinion publique n'était pas fiable dans son soutien au gouvernement ${ }^{111}$. David Eastwood admettait en revanche que «la menace la plus sérieuse pour l'ordre conservateur, dans les années 1790, serait venue d'une politisation de la détresse populaire par les radicaux ». Pourtant, comme I. Christie, D. Eastwood suggérait que l'accroissement très substantiel des poor rates ${ }^{112}$, accompagné d'initiatives comme la fondation en 1796 de la Société pour l'amélioration de la condition et l'accroissement du confort des pauvres, furent délibérément mises en place afin de combattre le problème politique de la pauvreté. Il ajoutait qu'elles furent efficaces ${ }^{113}$. À l'évidence, il est probable que la vérité se trouve quelque part entre les deux: les loyalistes actifs, convaincus et dans une certaine mesure convaincants dans leur utilisation de la propagande conservatrice, ne représentaient guère qu'une minorité assez large. Ce qui était crucial, c'était moins le loyalisme de masse que le consentement de masse, suffisamment durable pour assurer une stabilité globale, émaillée de moments de tensions et de confrontations, mais aussi 
d'épisodes où s'exprimait un patriotisme de masse ${ }^{114}$. Pour certains, ce consentement a pu naître de l'ignorance, du manque d'intérêt, ou d'un sentiment de fragilité. Pour d'autres, il a pu être obtenu grâce à une amélioration économique ou une philanthropie employée à bon escient. D'autres enfin ont pu être suffisamment enthousiasmés par les victoires militaires ou navales, lorsqu'elles survinrent, ou bien encore convaincus ou intimidés par la propagande ou les manifestations conservatrices, et seraient donc restés passifs. L'opinion publique, en d'autres mots, était volatile : prête à protester lorsque le contexte économique était délicat, ou quand les victoires se faisaient attendre, mais tendant à montrer son soutien ou à rester calme aussi longtemps qu'aucune menace réelle d'insurrection ne se profilait. Cette passivité était sans doute en partie naturelle et en partie le résultat d'une politique économique et d'un travail de propagande délibérés de la part du gouvernement et de ses sympathisants loyalistes.

Conclusion

Depuis une vingtaine d'années, les historiens ont dévoilé la vitalité du conservatisme et du loyalisme dans toute la société britannique des années 1790. Ils ont certes débattu de leur importance et de leur force réelle mais, comme l'a remarqué D. Eastwood, les historiens de la Grande-Bretagne du XVIII siècle ont préféré travailler ensemble, débouchant sur des résultats fructueux, plutôt que d'opter pour des positions extrêmes qui engendraient des débats virulents ${ }^{115}$. Pendant cette période, beaucoup d'informations ont été collectées sur la manière dont le loyalisme et le conservatisme ont fait face à la Révolution française. Notre compréhension du loyalisme au niveau local, et ce dans des strates sociales bien plus vastes que les seules élites gouvernantes, s'en est trouvée accrue. Il n'en reste pas moins que ce type d'études régionales et locales détaillées, pour l'Écosse, est encore trop rare. Plusieurs historiens ont soulevé la question de savoir comment l'élite gouvernante a pu admettre l'idée d'une mobilisation d'une citoyenneté active sans pour autant disposer de garanties sur sa loyauté. La réponse à cette question est encore incertaine, sans doute parce que ces deux perspectives n'étaient pas forcément réconciliées dans l'esprit des puissants. Le problème continua à susciter une vive appréhension chez ces derniers, lorsqu'il fallut recourir aux associations loyalistes et plus tard aux Volontaires pour combattre la menace française. À la suite de cela, le gouvernement supprima discrètement son soutien à ces organisations et cessa de tolérer d'autres intrusions populaires dans la vie politique ${ }^{116}$.

\section{NOTES}

1.Edmund BURKE, An Appeal from the New to the Old Whigs, in The Works of Edmund Burke, Bohn, London, 1883, III, 85.

2.H.T. DickINSON, Liberty and Property: Political Ideology in Eighteenth-Century Britain, Methuen, London, 1977, ch. 8.

3.Gayle TRUSDEL PENDLETON, «English Conservative Propaganda During the French Revolution, 1789-1802», non publié Ph.D. (Emory University, 1976). 
4.Nancy UHLAR MURRAY, «The Influence of the French Revolution on the Church of England and its Rivals, 1789-1802», Ph.D. non publié (University of Oxford, 1975). 5.Austin MiTCHELL, «The Association Movement of 1792-3», Historical Journal, 4,1961, pp. 56-77; E.C. Black, The Association: British Extra-Parliamentary Political Organization, 1769-1793, Oxford University Press, Oxford, 1963; Donald E. GINTER, «The Loyalist Association Movement of 1792-93 and British Public Opinion», Historical Journal, 9, 1966, pp. 179-190.

6.J. R. Western, «The Volunteer Movement as an Anti-Revolutionary Force, 1793-1801», English Historical Review, 71, 1956, pp. 603-614.

7.Par exemple, John MAccunN, The Political Philosophy of Edmund Burke, Edward Arnold, London, 1913 ; Alfred COBBAN, Edmund Burke and the Revolt against the Eighteenth Century, Allen \& Unwin, London, 1929; Charles PARKIN, The Moral Basis of Burke's Political Though, Cambridge University Press, Cambridge, 1956 ; Francis P. CANAVAN, The Political Reason of Edmund Burke, Cambridge University Press, Cambridge, 1960 ; R.R. FENNESSY, Burke, Paine and the Rights of Man, M. Nijhoff, The Hague, 1963 ; Carl B. ConE, Burke and the Nature of Politics, II: The French Revolution, University of Kentucky Press, Lexington, 1964 ; Gerald W. CHAPMAN, Edmund Burke. The Practical Imagination, Harvard University Press, Cambridge, Mass., 1967 ; Frank O'GoRmAn, Edmund Burke. His Political Philosophy, Allen \& Unwin, London, 1973 ; Michael FREEMAN, Edmund Burke and the Critique of Political Radicalism, Oxford University Press, 1980.

8.Mark PHILP, «Vulgar Conservatism, 1792-3», English Historical Review, 110, 1995, pp. 42-69; Kevin GILMARTIN, «In the Theater of Counterrevolution, Loyalist Association and Conservative Opinion in the 1790s ", Journal of British Studies, 41, 2002, pp. 291-328. 9.Tout particulièrement dans The Making of the English Working Class, Penguin, Harmondsworth, 1963, 1980, p. 12.

10.Voir les notes 43 et 44 .

11.GILMARTIN, «In the Theater of Counterrevolution», p. 296; voir aussi Mark PHILP, «Introduction », The French Revolution and British Popular Politics, Cambridge University Press, Cambridge, 1991, ed. Philp, p. 16.

12.The Correspondence of Edmund Burke, ed. Thomas W. Copeland et al., 10 vols., Cambridge University Press, Cambridge and Chicago, 1958-70.

13.Les deux volumes auxquels se réfère le présent article sont The Writings and Speeches of Edmund Burke, ed. Paul LANGFORD, vol. VIII: The French Revolution, ed. L.G. Mitchell, Oxford University Press, Oxford, 1989, et le vol. IX: Part 1. The Revolutionary War, 1794-1797; Part 2. Ireland, ed. R.B. McDowell, Oxford University Press, Oxford, 1991. 14.F.P. Lock, Burke's Reflections on the Revolution in France, Allen \& Unwin, London, 1985; et J.C.D. CLARK, Edmund Burke: «Reflections on the Revolution in France», Stanford, CA, 2001. 15.Alfred COBBAN, dans Edmund Burke and the Revolt Against the Eighteenth Century, Allen \& Unwin, London, 1929.

16.F.P. Lock, Edmund Burke, vol. 1, 1730-1784, Oxford University Press, Oxford, 1998. 17.Idem, Burke's Reflections on the Revolution in France, Allen \& Unwin, London, 1985, pp. 31, 29-30.

18. Nicholas K. ROBINSON Edmund Burke: A Life in Caricature, Yale University Press, New Haven, 1996.

19.Voir par exemple Peter STANLIS, Edmund Burke and the Natural Law, University of Michigan Press, Ann Arbor, 1958. 
20.John WHALE, «Introduction», in Edmund Burke's Reflections on the Revolution in France. New Interdisciplinary Essays, ed. John WHALE, Manchester University Press, Manchester, 2000, p. 12. Voir aussi Lock, Burke's Reflections on the Revolution in France, op. cit., pp. 88, 98-99.

21.J.G.A. Pocock, «The Political Economy of Burke's Analysis of the French Revolution», HJ, 25, 1982, pp. 331-349; idem, «Edmund Burke and the Redefinition of Enthusiasm: the Context of Counter-Revolution», dans The French Revolution and the Creation of Modern Political Culture, vol. III: The Transformation of Political Culture, 1789-1848, ed. François FURET and Mona OZOUf, Oxford University Press, Oxford, 1989, pp. 19-43.

22. Colin LUCAS, «Edmund Burke and the Émigrés », in The Transformation of Political Culture, 1789-1848, ed. FURET et OzOUF, pp. 101-114; Harvey MITCHELL, «Presentations», in ibid., pp. 3-18.

23.James K. CHANDLER, «Poetical Liberties: Burke's France and the "Adequate Representation" of the English», dans The Transformation of Political Culture, 1789-1848, ed. FURET et OzOUF, pp.49, 45-6.

24.Tom FURNISS, "Cementing the nation: Burke's Reflections on Nationalism and National Identity», dans Burke's Reflections, ed. WHALE, p.139.

25.Lock, Burke's Reflections, p. 164.

26.PHILP, «Vulgar Conservatism», p. 43.

27.Gregory CLAEYS, «Republicanism Versus Commercial Society: Paine, Burke and the French Revolution Debate», History of European Ideas, 11 (1989), p. 313; Kevin GILMARTIN, «Burke, Popular Opinion, and the Problem of a Counter-Revolutionary Public Sphere», in Burke's Reflections, ed. WHALE, pp. 94-95.

28.H.T. DiCKINSON, «Popular Conservatism and Militant Loyalism, 1789-1815», in Britain and the French Revolution, 1789-1815, ed. H.T. DICKINSON, Macmillan, Basingstoke, 1989, pp. 104-5; J.G.A. POCоCK, «Introduction», Reflections on the Revolution in France, Indianapolis, 1987, p. XL.

29.GILmARTIN, «Burke, Popular Opinion, and the Problem of a Counter-Revolutionary Public Sphere», pp. 99, 104.

30.Gregory CLAEYS, «The French Revolution Debate and British Political Thought», History of Political Thought, XI, 1990, pp. 59-80.

31.H.T. DiCKINSON, Liberty and Property: Political Ideology in Eighteenth-Century Britain, Methuen, London, 1977, ch. 8; idem, British Radicalism and the French Revolution 1789-1815, Blackwell, London, 1985; idem, «Popular Conservatism and Militant Loyalism 1789-1815», in Britain and the French Revolution 1789-1815, ed. DICKINSON, pp. 103-127; idem, «Popular Loyalism in Britain in the 1790s», in The Transformation of Political Culture. England and Germany in the Late Eighteenth Century, ed. Eckhart HELLMUTH, The German Institute, London, 1990, pp. 503-533; idem, «Britain and the Ideological Crusade Against the French Revolution», in Actes du Colloque international, ed. L. Domergue and G. Lamoine, Presses Universitaires du Mirail, Toulouse, 1992, pp. 153-74; idem, «The French Revolution and the Counter Revolution in Britain», in Aspekte der Französischen Revolution, ed. Hans-Christophe SCHRÖDER and Hans-Dieter METZGER, Technischen Hochschule Darmstadt, Darmstadt, 1992, pp. 231-263; idem, The Politics of the People in Eighteenth-Century Britain, St Martin's Press, Basingstoke, 1995, ch. 8. 32.DiCKINSON, «Popular Conservatism and Militant Loyalism», p.124. 33.Ibid., p. 104. 
34.Ian R. CHRISTIE, Stress and Stability in Late Eighteenth-Century Britain: Reflections on the British Avoidance of Revolution, Oxford University Press, Oxford, 1984. Voir aussi CHRISTIE, "Conservatism and Stability in British Society», in The French Revolution and British Popular Politics, ed. PHILP, pp. 169-87.

35.John DINWIDDY, «Interpretations of anti-Jacobinism», in The French Revolution and British Popular Politics, ed. PHILP, p. 38.

36.Linda COLLEY, «The Apotheosis of George III: Loyalty, Royalty and the British Nation», Past and Present, 102 (1984), pp. 94-129; Marilyn MORRIS, The British Monarchy and the French Revolution, Yale University Press, New Haven and London, 1998.

37.Thomas Philip SCHOFIELD, «English Conservative Thought and Opinion in Response to the French Revolution», non publié, Ph.D., University of London, 1984, p. 6; voir aussi idem, «Conservative Political Thought in Britain in Response to the French Revolution», Historical Journal, 29 (1986), pp. 601-622.

38.J.C.D. CLARK, English Society, 1688-1832, Cambridge University Press, Cambridge, 1985. 39.Michael DufFY, The Englishman and the Foreigner, Chadwyck-Healey, Cambridge, 1986; Stella M. Ní GHALLCHÓIR COTTRELL, «English Views of France and the French, 1789-1815», Ph.D. non publié, University of Oxford, 1991; idem, «The Devil on Two Sticks: francophobia in 1803», dans Patriotism: The Making and Unmaking of British National Identity, vol. 1: History and Politics, ed. Raphael SAMUEL, Routledge, London, 1991, pp. 259-74. Voir aussi Gerald NEWMAN, The Rise of English Nationalism: A Cultural History 1740-1830, Weidenfeld \& Nicolson, London, 1987, qui démontre bien l'ampleur de la gallophobie anglaise, même si, de façon surprenante, il n'utilise pas vraiment les sources qui abondent sur ce thème à partir des années 1790.

40.DINWIDDY, «Interpretations of anti-Jacobinism», pp. 45-46, 48-49.

41.J.E. CooKson, The British Armed Nation, 1793-1815, Oxford University Press, Oxford, 1997; Austin GEE, The British Volunteer Movement 1794-1814, Oxford University Press, Oxford, 2003.

42.Depuis quelques décennies, John Brims est à la pointe des travaux sur le radicalisme écossais des années 1790. Voir sa thèse de doctorat non publiée : «The Scottish Democratic Movement in the Age of the French Revolution», non publié, University of Edinburgh, 1983, qui contient aussi des informations utiles sur les conservateurs ; idem, "The Scottish "Jacobins", Scottish Nationalism and the British Union», in Scotland and England, 1286-1815, ed. Roger A. MASON, John Donald, Edinburgh, 1987, pp. 247-265; idem, «The Covenanting Tradition and Scottish Radicalism in the 1790s», in Covenant, Charter and Party. Traditions of Revolt and Protest in Modern Scottish History, ed. Terry BROTHERSTONE, Aberdeen University Press, Aberdeen, 1989, pp. 50-62; idem, «From Reformers to Jacobins: the Scottish Association of the Friends of the People», in Conflict and Stability in Scottish society, 1700-1850, ed. T.M. DEVINE, John Donald, Edinburgh, 1990, pp. 31-50; idem, «Scottish Radicalism and the United Irishmen», in The United Irishmen: Republicanism, Radicalism and Rebellion, ed. David DICKSON, Dáire KEOGH and Kevin WHELAN, Lilliput Press, Dublin, 1993. Voir aussi Elaine W. MCFARLAND, Ireland and Scotland in the Age of Revolution. Planting the Green Bough, Edinburgh University Press, Edinburgh, 1994. 43.Voir cependant David BRown, «Henry Dundas and the Government of Scotland», Ph.D. non publié, University of Edinburgh, 1989; idem, «The Government of Scotland under Henry Dundas and William Pitt», History, 83 (1998), pp. 265-279; et Michael FRY, The Dundas Despotism, Edinburgh University Press, Edinburgh, 1992, sur l'élite gouvernementale. 
44.Atle Libaek wOLD, «The Scottish Government and the French Threat, 1792-1802», Ph.O, University of Edinburgh, 2003.

45.Ibid., pp. 324-326.

46.Austin MitCHELL, «The Association Movement of 1792-3», Historical Journal, 4 (1961), pp. 56-77; E.C. BLACK, The Association: British Extra-Parliamentary Political Organization, 1769-1793, Oxford University Press, Oxford, 1963; Donald E. GINTER, «The Loyalist Association Movement of 1792-93 and British Public Opinion», Historical Journal, 9 (1966), pp. 179-190.

47.DOzIER, For King, Constitution and Country, pp. 48, 54-55.

48. Voir la note 26.

49.Alan Bоотн, «English Popular Loyalism and the French Revolution», in the Bulletin of the Society for the Study of Labour History, 54 (1989), p. 27; Idem, «Popular Loyalism and Public Violence in the North-West of England, 1790-1800», Social History, 8 (1983), p. 302. 50.DiCKINSON, «Popular Conservatism and Militant Loyalism», p. 119; idem, «Popular Loyalism in Britain», pp. 516-517; idem, Politics of the People, pp. 256, 284-286. 51.David EASTWOOD, «Patriotism and the English State in the 1790s», in The French Revolution and British Popular Politics, ed. Mark PHILP, Cambridge, 1991, not. p. 150. 52.Ibid., p. 155.

53.Michael DuFFY, «William Pitt and the Origins of the Loyalist Association Movement of 1792», Historical Journal, 39 (1996), pp. 943-962.

54.Mark PHILP, «Vulgar Conservatism, 1792-3», English Historical Review, 110 (1995), pp. 42-69; GILMARTIN, «In the Theater of Counterrevolution», Journal of British Studies, 41 (2002), pp. 291-328 (citation p. 295).

55.GILMARTIN, «In the Theater of Counterrevolution», p. 328. Le texte fait allusion à William PALEY, Reasons for Contentment. Addressed to the Labouring Part of the British Public (1792).

56.GEE, British Volunteer Movement, p. 4.

57. Voir Emma VINCENT MACLEOD, A War of Ideas: British Attitudes towards the War against Revolutionary France, 1792-1802, Ashgate, Aldershot, 1998, pp. 187-195.

58.J.R. WESTERN, «The Volunteer Movement as an Anti-Revolutionary Force, 1793-1801», English Historical Review, 71 (1956), pp. 603-614; DozIER, For King, Constitution and Country, pp. 138-139, 154; DiCKINSON, The Politics of the People, p. 282; voir aussi MACLEOD, A War of Ideas, pp. 70-72.

59.EASTWOOD, «Patriotism and the English State in the 1790s», op. cit., pp. 158-161;

COOKSON, British Armed Nation, ch. 3.

60.EASTWOOD, «Patriotism and the English state», op. cit., p. 159.

61.COOKSON, The British Armed Nation, pp. 73sq., 237sq.; cf. Linda COLLEY, Britons. Forging the Nation, 1707-1837, Yale University Press, New Haven, 1992, pp. 302, 305-308.

62.Idem, «The English Volunteer Movement of the French Revolutionary Wars, 1793-1815: Some Contexts», Historical Journal, 32 (1989), pp. 867-891; COLLEY, Britons, pp. 301-302; Paul LANGFORD, Public Life and the Propertied Englishman, Oxford University Press, Oxford, 1991.

63.GEE, British Volunteer Movement.

64.Ibid., p. 10.

65. MACLEOD, A War of Ideas, pp. 71-72.

66.Caledonian Mercury, 16 October 1794.

67. Caledonian Mercury, 3 January 1795. 
68.Ibid., 4 January 1798.

69.GEE, British Volunteer Movement, p. 264.

70.John BRIMS, «The Scottish Democratic Movement in the Age of the French Revolution», Ph.D. non publié, University of Edinburgh, 1983.

71.David BRown, «Henry Dundas and the Government of Scotland», Ph.D. non publié, University of Edinburgh, 1989.

72.Atle Libaek WOLD, «The Scottish Government and the French Threat, 1792-1802», Ph.D. non publié, University of Edinburgh, 2003.

73.Emma VINCENT MACLEOD, «A City Invincible? Edinburgh and the War against Revolutionary France», British Journal of Eighteenth-Century Studies, 23 (2000), pp. 153-166; Andrew MACKILLOP, «More Fruitful than the Soil»: Army, Empire and the Scottish Highlands, 1715-1815, Tuckwell Press, East Linton, 2000, ch. 7.

74. À paraître chez Birlinn: Edinburgh, pour Tuckwell Press.

75.Nancy U. MURRY, «The Influence of the French Revolution on the Church of England and its Rivals, 1789-1802», Ph.D. non publié, University of Oxford, 1975. Sur les conflits qui se déclenchèrent autour des Test and Corporation Acts avant la Révolution française, et une explication des peurs de l'Église par rapport à ses relations avec l'État à cet égard, voir aussi G.M. DiTCHFIELD, «The Parliamentary Struggle over the Repeal of the Test and Corporation Acts, 1787-1790», English Historical Review, 89 (1974), pp. 551-577. 76.Deryck LOVEGROVE, «English Evangelical Dissent and the European Conflict 1789-1815», Studies in Church History, 20 (1983), pp. 263-276.

77.David Hempton, Methodism and Politics in British Society, Hutchinson, London, 1984 voir pp. 57-58.

78.William STAFFORD, «Religion and the Doctrine of Nationalism in England the Time of the French Revolution and Napoleonic Wars», Studies in Church History, 18 (1982), pp. 381-395. Voir aussi M.J.D. ROBERTS, «Making Victorian Morals? The Society for the Suppression of Vice and its Critics, 1802-86», Historical Studies, 21 (1981), pp. 157-173; «The Society for the Suppression of Vice and its Early Critics, 1802-1812», Historical Journal, 26 (1983), pp. 159-176; Joanna INNES, «Politics and Morals: The Reformation of Manners Movement in Late Eighteenth-Century England», in The Transformation of Political Culture. England and Germany in the Late Eighteenth Century, ed. Eckhart HELLMUTH, The German Institute, London, 1990, pp. 57-118.

79.Robert Hole, Pulpits, Politics and Public Order in England, 1760-1832, Cambridge University Press, Cambridge, 1989; idem, «English Sermons and Tracts as Media of Debate on the French Revolution 1789-99», in The French Revolution and British Popular Politics, Cambridge University Press, Cambridge, 1991, ed. PHILP, pp. 18-37. Ces deux historiens traitent des écrits du clergé dans l'ensemble de l'échiquier politique. 80.HoLE, «English Sermons and Tracts», pp. 24, 31-32; idem, Pulpits, Politics and Public Order, p. 102.

81.Richard B. SHER, Church and University in the Scottish Enlightenment: the Moderate Literati of Edinburgh, Edinburgh University Press, Edinburgh, 1985, pp. 208, 305.

82.Ibid., pp. 209-210.

83.John R. MCINTOSH, Church and Theology in Enlightenment Scotland: The Popular Party, 1740-1800, Tuckwell Press, East Linton, 1998, pp. 227-228.

84.Emma VINCENT, «The Responses of Scottish Churchmen to the French Revolution, 1789-1802», Scottish Historical Review, 73 (1994), p. 205.

85. Voir aussi MACLEOD, A War of Ideas, ch. 6. 
86.Gayle TRUSDEL PENDLETON, «English Conservative Propaganda during the French Revolution, 1789-1802», non publié Ph.D., Emory University, 1976, p. 256.

87.Herbert M. ATHERTON, «The British Defend Their Constitution in Political Cartoons and Literature», in Studies in Eighteenth-Century Culture, vol. 11, Press of the Case Western Reserve University, Madison and London, 1982, ed. Harry C. PAYNE, pp. 3-31. Cf. Mary Dorothy GEORGE (ed.), Catalogue of Political and Personal Satires Preserved in the Department of Prints and Drawings in the British Museum, 7 vols., British Museum, London, 1935-54; idem, «Pictorial propaganda, 1793-1815: Gillray and Canning», History, 31 (1946), pp. 9-25; idem, "America in English Satirical Prints'», William and Mary Quarterly, 3rd series, 10 (1953), pp. 511-537; idem, English Political Caricature, 2 vols., Clarendon Press, Oxford, 1959.

88.DICKINSON, Politics of the People, p. 269.

89.Thomas Philip SCHOFIELD, «English Conservative Thought and Opinion in Response to the French Revolution 1789-1796», non publié Ph.D., University of London, 1984; idem, «Conservative Political Thought in Britain in Response to the French Revolution», Historical Journal, 29 (1986), pp. 601-622; idem, «British Politicians and French Arms: The Ideological War of 1793-5», History, 77 (1992), pp. 183-201.

90.Emily LORRAINE DE MONTLUZIN, The Anti-Jacobins 1798-1800: The Early Contributors to the "Anti-Jacobin Review », Macmillan, London, 1988.

91.Ibid., pp. 2, 26, 30-32, 35. Sur John Bowles, voir aussi Emma VINCENT, «"The Real Grounds of the Present War": John Bowles and the French Revolutionary Wars 1792-1802», History, 78 (1993), pp. 393-420.

92.Robert HOLE, «British Counter-revolutionary Popular Propaganda in the 1790s», in Britain and Revolutionary France, Exeter University Press, Exeter, ed. Colin JONES, pp. 53-69.

93.Ibid., p. 66.

94.M.O. GRENBY, The Anti-Jacobin Novel. British Conservatism and the French Revolution, Cambridge University Press, Cambridge, 2001. Voir l'article de Matthew GrenBY dans ce même numéro.

95.Jeremy BLACK, The English Press in the Eighteenth Century, Croon Helm, London, 1987; idem, The English Press 1621-1861, Stroud, 2001; Bob HARRIS, Politics and the Rise of the Press. Britain and France, 1620-1800, Routledge, London, 1996; Hannah BARKER, Newspapers, Politics and Public Opinion in Late Eighteenth-Century England, Oxford University Press, Oxford, 1998.

96.Hannah BARKER, Newspapers, Politics and English Society 1695-1855, Longman, Harlow, 2000, p. 176.

97.Bob HARRIs, «Scotland's Newspapers, the French Revolution and Domestic Radicalism (c.1789-1794)», Scottish Historical Review, 84, April 2005, p. 38-62, et aussi idem, «Print and Politics», in Scotland and the French Revolution, ed. Bob HARRIS, Birlinn, Edinburgh, 2005. Voir aussi, John Dwyer, «The Caledonian Mercury and Scottish National Culture, 1763-1801», in Politics and the Press in Hanoverian Britain, ed. Karl SCHWEIZER and Jeremy BLACK, Lewiston, 1989, pp. 147-169. Sur la presse des années 1790 plus généralement, voir aussi HARRIS, Politics and the Rise of the Press, pp.37-52, passim. 98.HARRIS, «Scotland's Newspapers», p. 54-55.

99.Michael DUFFY, The Englishman and the Foreigner, Chadwyck-Healey, Cambridge, 1986; H.T. DICKINSON, Caricatures and the Constitution, Chadwyck-Healey, Cambridge, 1986.

100.DICKINSON, op. cit., pp. 24-25; DUFFY, op. cit., p. 38. 
101.Diana DonALD, The Age of Caricature: Satirical Prints in the Age of George III, Yale University Press, New Haven and London, 1996, pp. 147-157; David BINDMAN, «Introduction», The Shadow of the Guillotine: Britain and the French Revolution, British Museum Publications, London, 1989, ed. David BINDMAN, Aileen DAWSON et Mark JONES, pp. 33-34.

102.Alexandra FRANKLIN et Mark PHILP, Napoleon and the Invasion of Britain, The Bodleian Library, Oxford, 2003.

103.Eirwen C. NICHOLSON, «English Political Prints and Pictorial Political Argument $c$. 1640-c.1832: a study in historiography and methodology", Ph.D. non publié, University of Edinburgh, 1994, ch. 9. Cf idem, «Consumers and Spectators: The Public of the Political Print in Eighteenth-Century England», History, 81 (1996), pp. 5-21. 104.Timothy Clayton, The English Print, 1688-1802, Yale University Press, New Haven, 1997, p. 232.

105.Robert L. PATTEN, George Cruikshank's Life, Times and Art, vol. 1: 1792-1835, Lutterworth Press, Cambridge, 1992, vol. 2, Lutterworth Press, Cambridge, 1996; David ALEXANDER, Richard Newton and English Caricature in the 1790s, Manchester University Press, Manchester, 1998; Richard GODFREY, James Gillray and the Art of Caricature, Tate Publishing, London, 2001; Nicholas ROBINSON, Edmund Burke: A Life in Caricature, Yale University Press, New Haven and London, 1996; Vincent CARRETTA, George III and the Satirists From Hogarth to Byron, University of Georgia Press, Athens, Georgia and London, 1990.

106.Mark PHILP, «The Fragmented Ideology of Reform», p. 70.

107.Ibid., pp. 52-59.

108. Une accusation contemporaine des partisans de Fox, enterrée de façon probante par Clive Emsley au début des années 1980 dans deux articles : «An Aspect of Pitt's Terror: Prosecutions for Sedition during the 1790s», Social History, 6 (1981), pp. 155-184; idem, «Repression, "terror" and the Rule of Law in England during the Decade of the French Revolution», English Historical Review, 100 (1985), pp. 801-825.

109.Mark PHILP, «Introduction», The French Revolution and British Popular Politics, ed. PHILP, p. 13.

110.Ian R. CHRISTIE, Stress and Stability in Late Eighteenth-Century Britain: Reflections on the British Avoidance of Revolution, Oxford University Press, Oxford, 1984; idem, "Conservatism and Stability in British Society», in The French Revolution and British Popular Politics, ed. Mark PHILP, pp. 169-187.

111. Roger WELLS, Wretched Faces: Famine in Wartime England, 1793-1801, Alan Sutton, Gloucester, 1988; idem, Insurrection: The British Experience, 1798-1803, Alan Sutton, Gloucester, 1983 ; idem, «English Society and Revolutionary Politics in the 1790s: the Case for Insurrection», in The French Revolution and British Popular Politics, ed. PHILP, pp. 188-226.

112.NdT. Impôt local payé par les propriétaires de chaque paroisse pour couvrir le coût de l'assistance aux pauvres.

113.EASTWOOD, «Patriotism and the English State», pp. 162-165.

114.See MACLEOD, A War of Ideas, ch. 8.

115.David EASTWOOD, compte rendu de Dickinson, Politics of the People, in British Journal for Eighteenth-Century Studies, 20 (1997), p. 86.

116. Cookson, The British Armed Nation, ch. 3; Gee, British Volunteer Movement, ch. 7 et conclusion. 


\section{RÉSUMÉS}

Dans les deux dernières décennies, les écrits en langue anglaise portant sur la réaction des conservateurs britanniques face à la Révolution française ont étudié le loyalisme britannique à l'échelle locale, et dans des couches sociales bien plus vastes que les seules élites gouvernantes. La question soulevée est celle de savoir comment l'État britannique a autorisé et même encouragé les classes moyennes et populaires à prendre part à la défense de sa constitution élitiste.

The problem of the active citizen: conservative reactions to the French Revolution in Britain. Writings in English over the last two decades on the conservative reaction in Britain to the French Revolution have examined British loyalism at a local level and in social strata well below those of the governing elite. They have explored the issue of how the British state allowed and even encouraged the lower and middle orders to participate in defending its elitist constitution.

INDEX

Mots-clés : volontaires, propagande, religion, Burke, loyalistes, conservateurs

\section{AUTEUR}

EMMA VINCENT MACLEOD

University of Stirling, History Department, Stirling, FK9 4LA, Écosse, Royaume-Uni 